\title{
Plasmon-enhanced Electron Harvesting in Robust Titanium Nitride Nanostructures
}

\author{
Brock Doiron ${ }^{\mathrm{A}}$, Yi Li ${ }^{\mathrm{D}}$, Andrei Mihai ${ }^{\mathrm{B}}$, Ryan Bower ${ }^{\mathrm{B}}$, Neil McN. Alford ${ }^{\mathrm{B}}$, Peter K. Petrov ${ }^{\mathrm{B}}$, Stefan A. \\ Maier ${ }^{\mathrm{D}, \mathrm{A}}$, Rupert F. Oulton ${ }^{\mathrm{A}}$ \\ A Department of Physics, Imperial College London, London, UK \\ B Department of Materials, Imperial College London, London, UK \\ D Nanoinstitut München, Fakultät für Physik, Ludwig-Maximilians Universität München, München, \\ Germany
}

\section{SUPPLEMENTARY INFORMATION}

Contents: 4 pages, 4 sections, 6 figures 


\section{$\underline{\text { S1 FDTD Simulated Electric Field Intensity }}$}
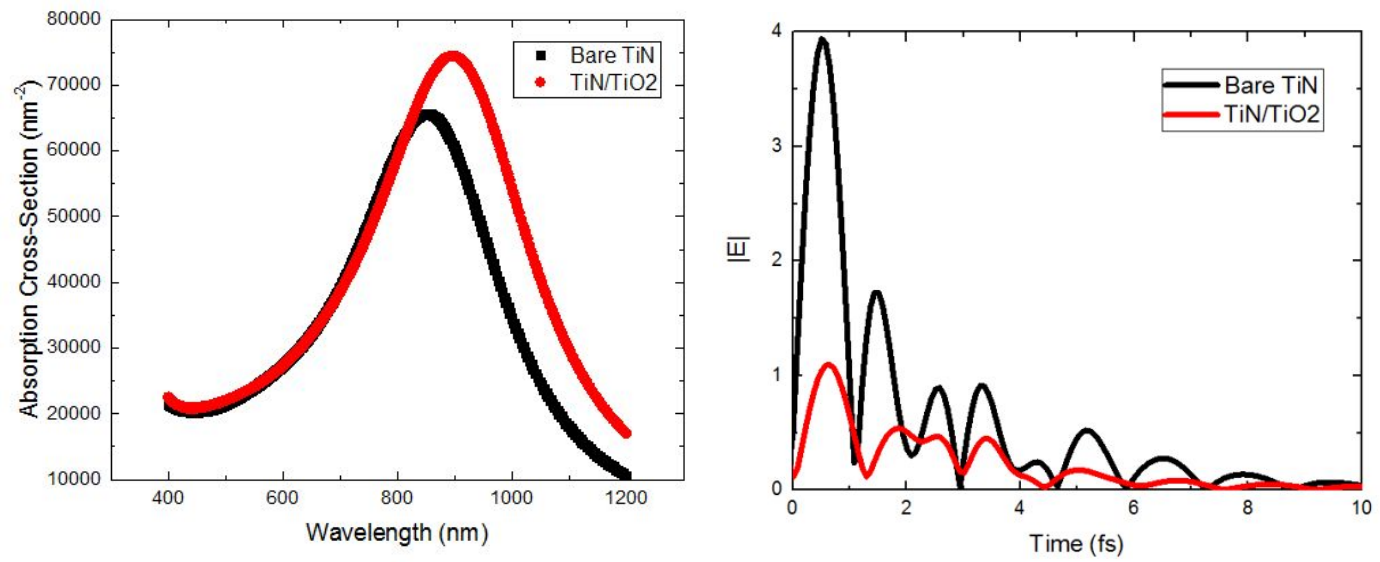

Figure S1 | (Left) Simulated absorption cross-section of TiN strips (150 nm x 30 um) with and without an encapsulating surface oxide layer $(5 \mathrm{~nm})$ showing an increase in magnitude, red-shift and broadening consistent with plasmon damping. (Right) FDTD simulations of temporal evolution of the electric field intensity at the surface of a $\mathrm{TiN}$ disk with and without a $\mathrm{TiO}_{2}$ surface oxide layer.

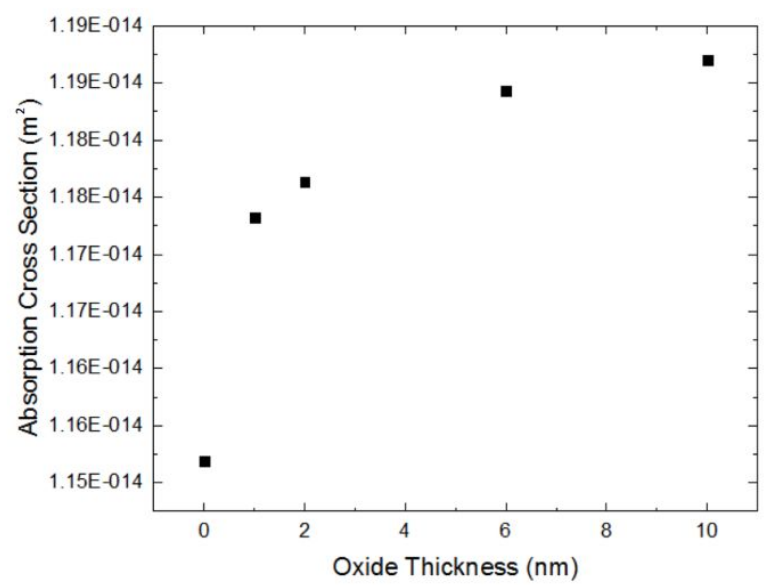

Figure S2 | Variation of absorption of a TiN disk with increasing oxide thickness simulated by FDTD. There is a rapid change between no oxide $(0 \mathrm{~nm})$ and $1 \mathrm{~nm}$ thick due to the change in refractive index and then a slowerincrease up to $10 \mathrm{~nm}$.

\section{$\underline{\text { S2 Trap-Assisted Recombination at a Metal-Semiconductor Interface }}$}

The model used to fit the experimental transient pump-probe data was formulated in detail in our previous work. ${ }^{1}$ The evolution of free carrier concentration $\left(N_{F C}\right)$ is described by two relatively shorttimescale processes (trapping into deep or shallow traps) and one long-timescale process (recombination with holes back in the metal). Quantifying the occupation of shallow and deep traps by $N_{S h}$ and $N_{D e}$ the free carrier concentration is described by:

$$
N_{F C}(t)=N_{F C}(0)-N_{S h}(t)-N_{D e}(t)
$$

Eventually, a quasi-equilibrium is reached between trap occupation and recombination and the decrease in differential reflectivity $\left(N_{F c}\right)$ plateaus. Solving the associated differential reflections gives rise to a bi-exponential behaviour: 


$$
\frac{\Delta R}{R}=\delta+s e^{-t / \tau_{S h}}+d e^{-t / \tau_{D e}}
$$

Where $s$ and $d$ are the percent of initial energy lost to shallow and deep traps and $\tau_{S h}$ and $\tau_{D e}$ are the associated free electron trapping lifetimes (inverse of the electron trapping rates). Any physical change to the density of trap states or the frequency on electron-trap interaction (electron conductivity) will be reflected by changes in these parameters. An increase in trap density will result in more electrons losing their energy to traps and an increase in the amplitudes ( $s$ and/or $d$ ). As seen below we see a possible decrease in deep trap density post-annealing by fitting the pump probe data to the model above.
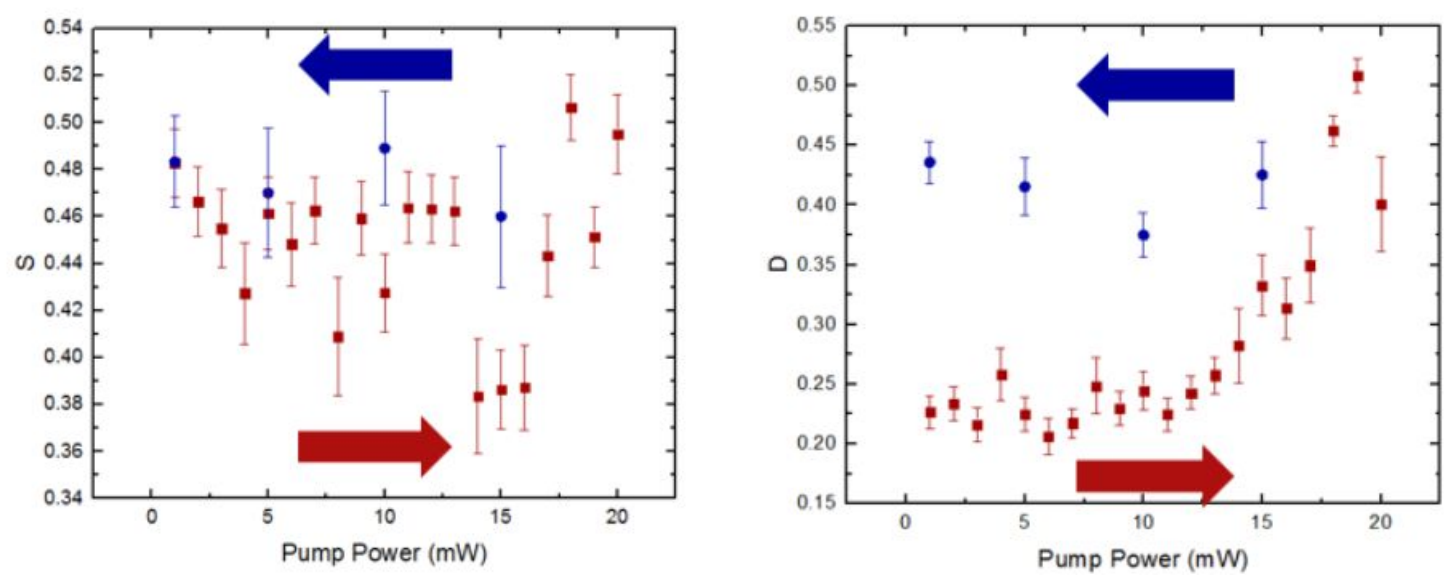

Figure S3 | Fitted exponential amplitudes of shallow (left) and deep (right) trapping during heating (red) and cooling (blue) as described by the trap-mediated recombination model. This serves as an estimate of the energy lost to each pathway. Annealing at high laser powers permanently alters the deep trap density whereas no change is observed in the shallow traps.

(1) Doiron, B.; Yi Li; Andrei Mihai; Stefano Dal Forno; Sarah Fearn; Lesley F Cohen; Neil M Alford; Johannes Lischner; Peter Petrov; Stefan A Maier; Rupert Oulton. Optimizing Hot Electron Harvesting at Planar Metal-Semiconductor Interfaces with Titanium Oxynitride Thin Films. ArXiv 2018, 1-21.

\section{S3 Pump Wavelength Sweep}

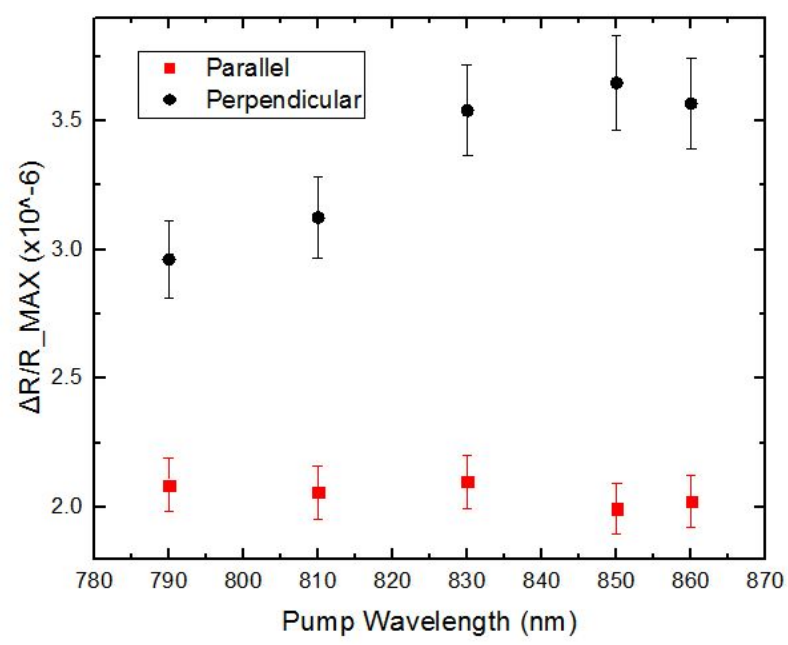


Figure S4 | Maximum differential reflectivity versus pump wavelength at both parallel-polarized (non-resonant) and perpendicular-polarized (resonant) excitation showing the clear dependence on surface plasmon excitation.
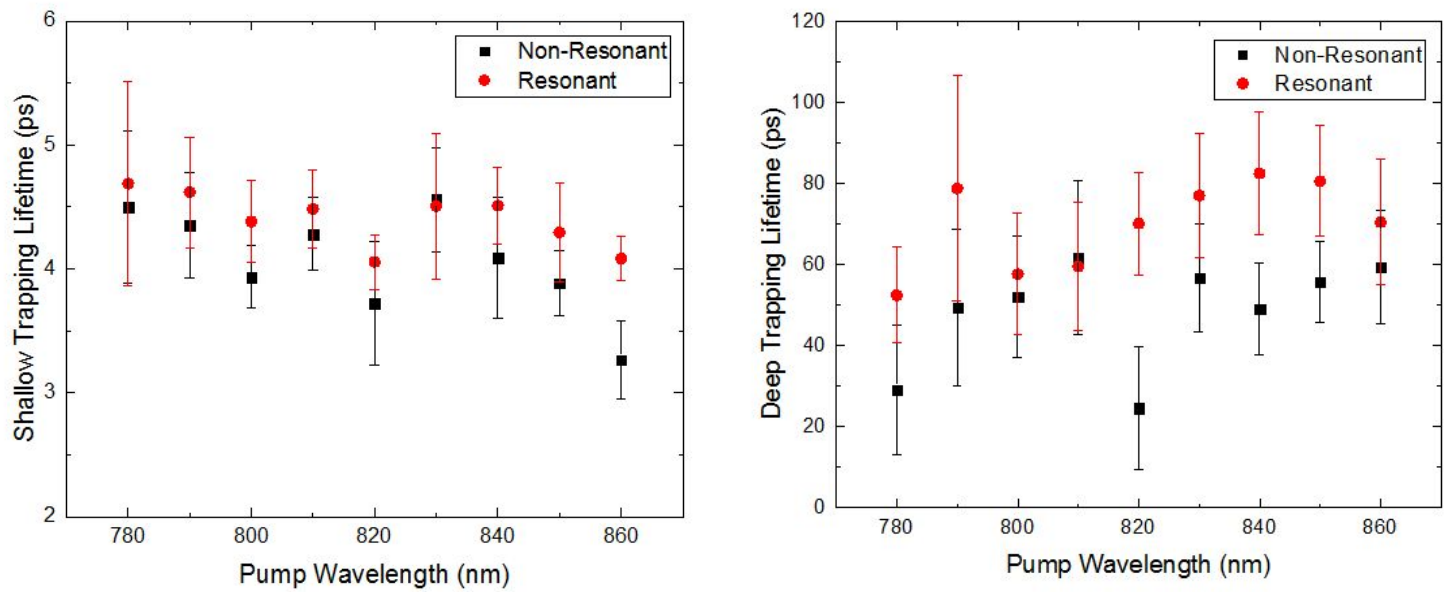

Figure S5 | Shallow and deep trapping lifetimes excited in non-resonant and resonant conditions using the model described in Supplementary Section S2. No significant dependence on the pump wavelength suggests that the measured quantities are material-dependent.

\section{S4 X-Ray Diffraction of TiN films}
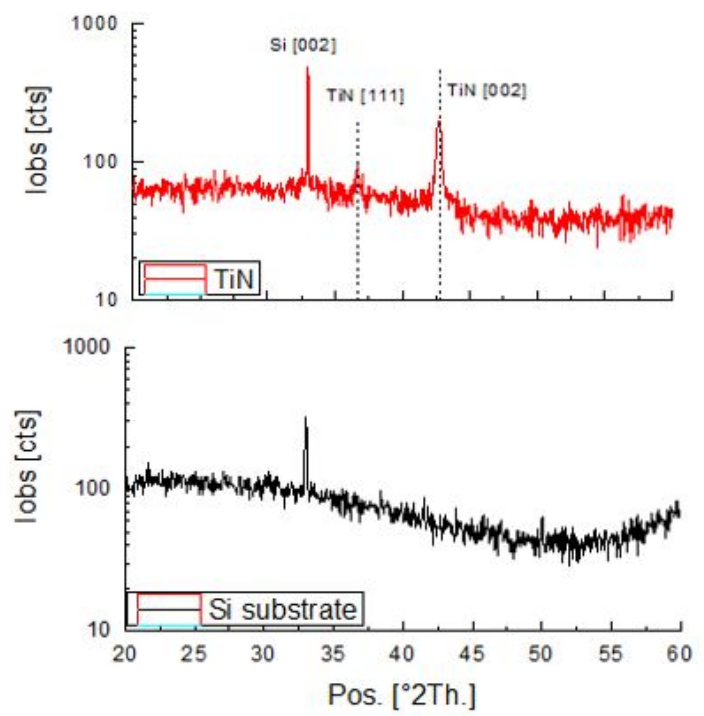

Figure S6 | X-Ray Diffraction measurements of TiN deposited on a Si substrate as well as the bare Si substrate. The TiN [002] is the strongest with the TiN[111] peak still visible. 